posits and the "full" house immunofluorescence in our case do show some similarities to lupus nephritis. However, clinical examination did not suggest coexistent SLE; antinuclear antibodies were not measured.

There was also particularly strong staining for IgA, which was only described in one of the Finnish patients. Schönlein Henoch purpura is associated with IgA deposition and could produce this type of renal lesion. It is notable that a petechial rash was noticed terminally in our patient and that there were multiple haemorrhages over the serosal aspect of the bowel, but these could be secondary to the coexistent coagulopathy.

The liver plays a central role in clearance of immune complexes from the circulation and there is a well documented association between liver cirrhosis and a glomerulopathy characterised particularly by mesangial immune complexes and IgA deposition. ${ }^{6}$ The features described in this glomerulopathy are very sim- ilar to those seen in the current case; it may be that the glomerulopathy in LPI is related to concomitant liver injury rather than representing a distinctive lesion.

The authors wish to thank Mr J Sandford, Mr Roy Creighton and $\mathrm{Mr}$ Thomas McLoughlin for technical assistance.

Originally presented as a poster presentation at the 170th Meeting of The Pathological Society of Great Britain and Ireland.

1 Simell O. Chapter 18. Lysinuric protein intolerance and other cationic amino-acidurias. In: Scriver CR, ed. Lysinuric protein intolerance and other cationic aminoacidurias. The metabolic and molecular basis of inherited disease. New York: McGraw Hill, 1995:3603-27.

2 Parto K, Kallajoki M, Aho H, Simell O. Pulmonary alveolar proteinosis and glomerulonephritis in lysinuric protein intolerance: case reports and autopsy findings of four paediatric patients. Hum Pathol 1994;25:400-7.

3 Carson NAJ, Redmond OAB. Lysinuric protein intolerance. Ann Clin Biochem 1977;14:135.

4 Cotran RS, Kumar V, Robbins SL. Chapter 1. In: Cellular injury and adaptation. Pathologic basis of disease. 4th edn. Philadelphia: WB Saunders, 1989:21.

5 Nagata M, Suzuki M, Kawamura G, Kono N, Yamaguchi $\mathrm{S}$, Aoki K. Immunological abnormalities in a patient with lysinuric protein intolerance. Eur F Pediatr 1987;146:427-8. 6 Emancipator SN, Heptinstall RH. Chapter 6. In: IgA nephritis and Schonlein Henoch syndrome. Pathology of the kidney. 4th edn. Boston: Little Brown, 1992:443-51.

\title{
Intimal sarcoma of the right brachiocephalic vein presenting as the superior vena caval syndrome
}

\author{
E R C Rytina, Y K Govil, K Sabanathan, R Y Ball
}

\section{Departments of Histopathologyl Cytopathology, Norfolk \& Norwich Health Care NHS Trust, Brunswick Road, Norwich NR1 3SR E R C Rytina R Y Ball}

Medicine for the Elderly

Y K Govil

K Sabanathan

Correspondence to: Dr R Y Ball.

Accepted for publication 11 September 1995

\begin{abstract}
A case of an 84 year old man presenting with obstruction of the superior vena cava caused by an intimal sarcoma of the right brachiocephalic vein is reported. The tumour morphology was similar to intimal sarcomas arising in major arteries, a more common primary site, and showed malignant fibrous histiocytoma-like features. Immunohistochemistry was suggestive of myofibroblastic differentiation.

(f Clin Pathol 1996;49:347-349)
\end{abstract}

Keywords: intimal sarcoma, vein, superior vena caval syndrome.

Primary sarcomas of the great vessels are rare and usually arise in the aorta, inferior vena cava, or pulmonary artery. ${ }^{1}$ Sarcomas of the thoracic veins are of exceptional rarity and may be associated with the superior vena caval syndrome. ${ }^{12}$ Here, we describe a primary intimal sarcoma of the right brachiocephalic vein which presented in this manner.

\section{Case report}

An 84 year old man complained of increasing breathlessness, occasional bloodstaining of sputum, and worsening headache of one month's duration. He had had a cough, productive of mucoid sputum, for two years. The past medical history included: appendicitis; partial gastrectomy for perforated duodenal ulcer; emphysema; cervical spondylosis; prostatic resection for nodular hyperplasia; and inguinal herniorrhaphy. $\mathrm{He}$ was alert, orientated and thin, seemed to have lost weight, and showed central cyanosis, suffusion of the face, and conjunctival congestion and oedema. The neck veins were engorged to the mandibular angle and showed no pulsation. Those over the chest were dilatated and their flow was towards the abdomen. There was bronchial breathing with increased vocal resonance and some crepitations in the right infraclavicular region. Small hard mobile lymph nodes were palpable in both axillae. No other significant findings were made on clinical examination.

Chest $x$ ray showed emphysematous lung fields, possible enlargement of the right paratracheal lymph nodes and no evidence of mediastinal masses or lung tumours. A computed tomography scan of the neck and chest (fig 1) showed a mass filling the lumen of the right brachiocephalic and internal jugular veins with evidence of obstruction of the left brachiocephalic vein and superior vena cava. 


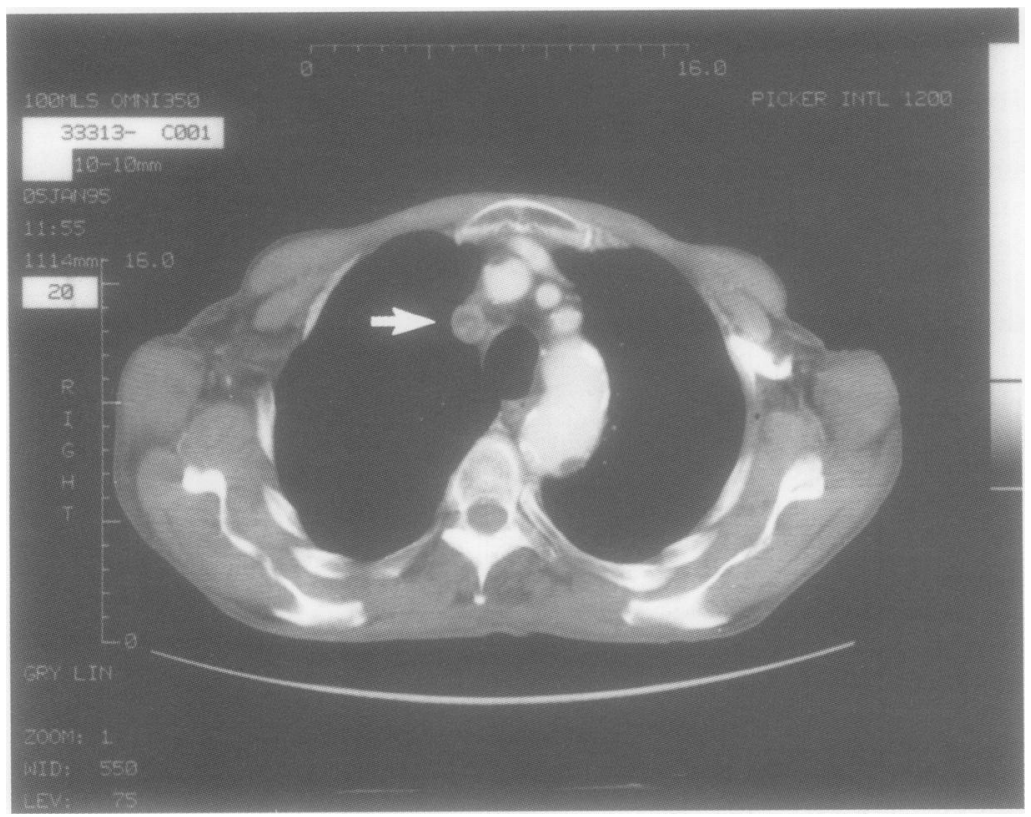

Figure 1 Computed tomography scan of the upper mediastinum showing a mass filling the lumen of the right brachiocephalic vein (arrow).
There was a lymph node mass in the pretracheal and precarinal regions. Sputum cytology was negative for tumour cells and serum tumour markers (Ca19-9; prostate specific antigen) were negative. Biopsy of a left axillary lymph node showed no evidence of malignancy. Ultrasound scan of the abdomen and pelvis was normal.

The patient was treated with radiotherapy (total radiation dose $1602 \mathrm{cGy}$ in two fractions over seven days) but the response was poor. He developed bronchopneumonia and died several days after stopping this treatment.

Postmortem examination showed a fleshy grey tumour arising within the right brachiocephalic vein (fig 2). It filled the lumen and extended into the lower part of the right internal jugular vein and the upper part of the superior vena cava. The tumour showed slight focal extension into the soft tissues between the vein and trachea. The right internal jugular vein above the tumour was occluded by thrombus. Much of the superior vena cava and the left brachiocephalic and internal jugular veins appeared unremarkable. There was no obvious local or distant soft tissue origin for the tumour. An anterior tracheal lymph node was considerably enlarged $(3.5 \mathrm{~cm}$ in diameter) by a metastasis. Distant metastases were not recognised.

Microscospy confirmed the tumour to be a sarcoma arising in the intima of the right brachiocephalic vein. It invaded the innermost media focally and extended along small tributaries. The tumour had a fine reticulin pattern forming groups of pleomorphic spindle-shaped cells, epithelioid cells plus bizarre multinucleate giant cells and contained a dense focal infiltrate of neutrophil polymorphs, lymphocytes and plasma cells. Many small vessels were present in the tumour and there was focal necrosis. Stains for mucins were negative. The tumour cells were negative in immunohistochemical studies for cytokeratins, desmin, desmin D33,

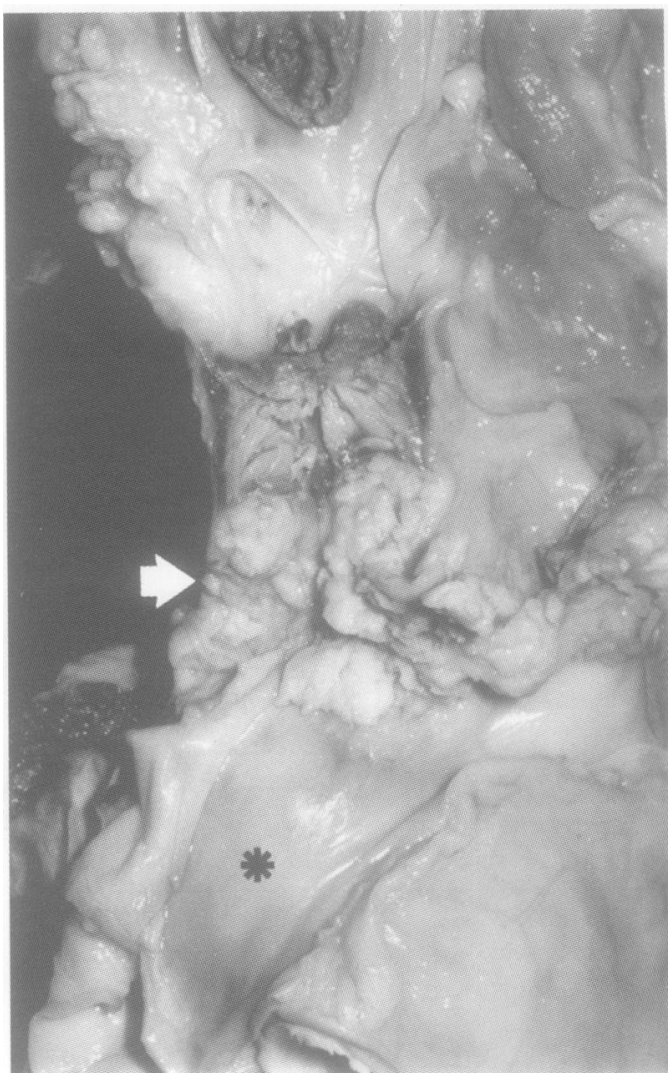

Figure 2 Intimal sarcoma of the right brachiocephalic vein (arrow) with thrombus in the vein above. *Superior vena cava.

factor VIII related antigen, CD31, and $\alpha-1-$ antitrypsin. They contained vimentin and some spindle-shaped cells expressed smooth muscle actin. Some of the cells, predominantly epithelioid in type, expressed nuclear and cytoplasmic S-100 protein. The appearances were those of an undifferentiated intimal sarcoma most closely resembling malignant fibrous histiocytoma (MFH). There was no dysplasia in the adjacent endothelium.

\section{Discussion}

Primary sarcomas arising in veins are rare, and origin in the brachiocephalic vein has not been reported before. Most are leiomyosarcomas, 15 out of 16 cases in one study, ${ }^{1}$ and they usually arise in the inferior vena cava. Intimal sarcomas most often arise in the pulmonary artery or aorta, ${ }^{1}$ there being only one reported case involving the superior vena cava. ${ }^{2}$ Burke and Virmani ${ }^{1}$ describe one case of intimal sarcoma of the inferior vena cava. Non-myogenic sarcomas are also seen in the pulmonary veins. ${ }^{34}$

Intimal sarcomas are histologically varied, ${ }^{15}$ tending to be undifferentiated with $\mathrm{MFH}$-like areas but sometimes showing angiosarcomatous, osteosarcomatous, or other patterns of differentiation. Pulmonary artery intimal sarcomas tend to be myxoid. ${ }^{1}$ The fact that most of these tumours occur in particular vessels (pulmonary artery, aorta) suggests they are distinct from those arising in the media, which usually affect different vessels.

Most reported venous intimal sarcomas have a similar microscopic appearance to tumours 
of arterial origin ${ }^{34}$ although one in the inferior vena cava also contained undifferentiated small cells ${ }^{1}$ and a case in the superior vena cava was epithelioid with only moderate pleomorphism. ${ }^{2}$ Intimal sarcomas show immunopositivity for vimentin, most are positive for smooth muscle actin, ${ }^{1}$ some for factor VIII related antigen ${ }^{267}$ and one for cytokeratin ${ }^{2}$ but all are negative for desmin. Burke and Virmani ${ }^{1}$ suggest that the tumours arise from intimal myofibroblastic cells and the immunohistochemistry in this case supports such a suggestion, markers of endothelium being negative. Factor VIII related antigen positivity and adjacent endothelial atypia have lead to the suggestion of a possible endothelial origin. ${ }^{67}$ However, in 10 cases of intimal sarcoma there was no factor VIII related antigen positivity. ${ }^{1}$

Here, we described an uncommon tumour, intimal sarcoma, arising in a previously unreported site, the right brachiocephalic vein. It had caused obstruction of the superior vena cava, an extremely rare cause of this syndrome. Most intimal sarcomas arise in arteries and show a variety of histological and immunohistochemical appearances. This venous tumour had features similar to most arterial neoplasms, showing evidence of myofibroblastic lineage and an MFH-like morphology. Despite palliative radiotherapy, survival after presentation was short, reflecting the generally poor prognosis of intimal sarcomas. ${ }^{1}$

We are grateful to Karen Turner for typing the manuscript and to members of the Department of Medical Illustration for the photographs.

1 Burke AP, Virmani R. Sarcomas of the great vessels. A clinicopathological study. Cancer 1993;71:1761-73.

2 Downes AJ, Jones TJ, Wilson RSE. Intimal sarcoma of the superior vena cava. Postgrad Med f 1993;69:155-6.

3 Kaiser LR, Urmacher C. Primary sarcoma of the superior pulmonary vein. Cancer 1990;66:789-95.

4 Khan MA, Davidson RC. Malignant fibrous histiocytoma of the pulmonary vein with prolapse through the mitral valve orifice. Postgrad Med F 1992;68:680-2.

5 Enzinger FM, Weiss SW (eds). Miscellaneous sarcomas of vascular origin. In: Soft tissue tumours. 3rd edn. St Louis: Mosby, 1995:508.

6 Fitzmaurice RJ, McClure J. Aortic intimal sarcoma: an unusual case with pulmonary vasculature involvement. Histopathology 1990;17:457-62.

7 Haber LM, Truong L. Immunohistochemical demonstration of the endothelial nature of aortic intimal sarcoma. $A m \mathcal{F}$ Surg Pathol 1988;12:798-802.

\title{
Alpha-fetoprotein production by a malignant mixed müllerian tumour of the uterus
}

\author{
K-A Phillips, J P Scurry, G Toner
}

\begin{abstract}
A case of $\alpha$-fetoprotein production by a uterine malignant mixed müllerian tumour is described. The patient was a 68 year old woman who developed intraabdominal recurrence of a stage 1 uterine tumour which had been treated surgically seven years previously. Her serum $\alpha$-fetoprotein was raised at $21000 \mu \mathrm{g} / \mathrm{l}$ (normal $<10 \mu \mathrm{g} / \mathrm{l})$ and staining with immunoperoxidase confirmed that the tumour was the site of $\alpha$-fetoprotein production. The patient was treated with combination chemotherapy but died two weeks after the first course. This is believed to be only the second such case reported.

(f Clin Pathol 1996;49:349-351)
\end{abstract}

Keywords: $\alpha$-fetoprotein, malignant mixed mesodermal tumour, uterus.

Alpha-fetoprotein is an important tumour marker in germ cell tumours and hepatocellular carcinoma and occasionally may be raised in other cancers. In 1985, Kawagoe reported a case in which a malignant mixed müllerian tumour (MMMT) produced $\alpha$-fetoprotein. ${ }^{1}$ Here, we report a second case and discuss the relevance to the histogenesis of MMMT and the possibility of using serum $\alpha$-fetoprotein concentrations to monitor the progress of disease.

\section{Case report}

A 68 year old woman presented with a one month history of symptoms of partial small bowel obstruction. A computed tomography scan showed a large heterogeneous intra-abdominal mass and a hyperechoic liver. She had a past history of a stage 1 uterine MMMT which had been treated by total hysterectomy, bilateral salpingo-oophorectomy and pelvic lymphadenectomy seven years previously and a pulmonary carcinoid tumour by left upper lobectomy six years later. Laboratory investigations included a noticeably raised serum $\alpha$-fetoprotein concentration $(21000 \mu \mathrm{g} / 1$ (normal $<10 \mu \mathrm{g} / \mathrm{l})$ ) and a raised CA 125 (710 units/l (normal <35 units/l)). The patient underwent a needle core biopsy of the abdominal mass.

\section{PATHOLOGY}

The core biopsy specimen, which measured $0.5 \times 0.1 \mathrm{~cm}$, showed metastatic MMMT with sharply demarcated carcinomatous and sar- 